\title{
Conflicts in Yemen exacerbate lost to follow-up rates of people living with HIV
}

\author{
Mayada Faisal Nabih M., ${ }^{1,2}$, Sharifa E. Wan Puteh², Amrizal Muhammad Nur² \\ ${ }^{1}$ National AIDS Control Program (NAP), Ministry of Public Health \& Population, Yemen \\ ${ }^{2}$ International Centre for Casemix and Clinical Coding (ITCC), Faculty of Medicine, Universiti Kebangsaan Malaysia (UKM)
}

\begin{abstract}
Introduction: The aim of the study is to examine demographic data of people living with human immunodeficiency virus (PLHIV) who are lost to follow-up (LTFU) during HIV treatment and care, in order to develop rapid and effective interventions to improve access to services in Yemen.
\end{abstract}

\begin{abstract}
Material and methods: This is a retrospective descriptive study of health service data implemented in all sites that provided HIV treatment and care services from 2007-2012. The study population was 1,586 PLHIV registered at pre-antiretroviral (pre-ART) clinics and 995 under antiretroviral (ART) clinics with exclusion of transfers and deaths.

Results: Most of the LTFU at pre-ART and ART clinics occurred during the conflict period (2011-2012) and among males at age 25-49 years, especially from governorates where the services existed. The first 3 years showed higher rates of LTFU; $90 \%$ and $81 \%$ were among Yemenis, and $68 \%$ and $65 \%$ among young males at pre-ART and ART respectively. Sana'a governorate had the highest rate of LTFU among females at pre-ART, while Aden experienced the highest rate of LTFU among females under ART.

Conclussion: The conflict in Yemen was associated with an increase in the rate of LTFU among Yemeni young males, especially at governorates that experienced political conflicts. In-depth study on LTFU are needed, and improved counselling methods and patient's tracking systems are recommended.
\end{abstract}

HIV AIDS Rev 2018; 17, 1: 49-53

DOI: https://doi.org/10.5114/hivar.2018.73349

Key words: lost to follow-up of PLHIV, Yemen conflicts, retention in care, antiretroviral therapy.

\section{Introduction}

Since 1981, acquired immune deficiency syndrome (AIDS) has killed more than 35 million people. It is estimated that 36.7 million people were living with human immunodeficiency virus (PLHIV) in the world. At the beginning of the epidemic, most of PLHIV died at first 10 years of infection [1]. In 1996, antiretroviral therapy (ART) made a revolution in changing AIDS diagnosis to chronic disease. However, the number of deaths among PLHIV in developing countries is still high due to limited resources, which make the treat-

Address for correspondence: Dr. Mayada Faisal Nabih M., Faculty of Medicine, International Centre for Casemix and Clinical Coding Universiti Kebangsaan Malaysia (ITCC-UKM),

e-mail: mayadanabih@yahoo.com

ment unaffordable. In 2003, a case of emergency situation has been announced in the world, and most of countries with limited resources and International Organizations implemented action plans to increase ART coverage. Therefore, more than a third of PLHIV who were in need of ART in these countries were able to get the treatment by the end of 2009 [2].

Since the ART does not cure HIV infection, PLHIV have to take treatment on a regular basis for the rest of their lives. But in some care and treatment programs, there are more than a third of patients lost to follow-up (LTFU) within the first three years from the start of ART [2]. LTFU in ART

Article history:

Received: 08.09.2017

Received in revised form: 06.10.2017

Accepted: 05.11.2017

Available online: 30.01 .2018
International Journal of HIV-Related Problems

HIV \& AIDS

R e vi e w 
period is define as an interval of 90 days or more, where PLHIV have missed their appointments at the ART clinic after last visit [3].

LTFU of PLHIV threatens the success of long-term treatment programs all over the world, and can cause serious consequences due to discontinuation of ART as well as increase risk of deaths. So, it is necessary to understand the underlying causes of LTFU [2].

Although there is a progress in HIV treatment in the last fifteen years, existing challenges does not ensure the continuity of ART and good attendance of PLHIV at HIV treatment and care clinics. There are various factors affecting access to health care services, which might be related either to PLHIV or to healthcare system [4].

The political commitment in Yemen toward national AIDS response is represented by the existence of National AIDS control Program (NAP) and National Strategic Plan for HIV and AIDS, where HIV treatment and care are considered as the main priority area [5]. However, there are only five locations established at government hospitals in five main governorates out of 22 governorates in Yemen (Sana'a, Aden, Taiz, Hodeida, and Mukalla) to provide treatment and care services for PLHIV, since 2007 by support of the Global Fund to fight AIDS, tuberculosis (TB), and malaria [6].

HIV epidemic in Yemen is driven mainly through sexual transmission in high-risk populations, especially female sex workers (FSW) and their clients, and men who have sex with men (MSM). According to 2011 HIV size estimates, the Country is having low HIV prevalence $(0.2 \%)$ among the general population. However, an evidence showed that Yemen have a concentrated HIV epidemic among MSM (6\%). Since 1987 till the end of 2012, the cumulative number of reported cases was 3,502 (71\% Yemenis, 63\% males). The estimated number of PLHIV in Yemen for the same period was 35,000 [7].

In fact, some countries in the Middle East and North Africa (MENA) have engaged into crisis that started decades ago, and has become worse over time [8]. Yemen is one of the countries in MENA region that faced intermittent conflict since 2011 , with $60 \%$ of people needing humanitarian assistance (by the end of 2014) due to poverty, underdevelopment, and environmental decline [9]. By September 2015, there was 2,204 civilian deaths, and 4,711 civilian injuries due to armed conflicts in March 2015 [10]. The conflict results in displacement of people due to collapse of social networks and institutions, exposing them to certain diseases including HIV, with no access to HIV services [11].

In Yemen, the number of new HIV cases reported in 2011 and 2012 was lesser comparing to the incidence in the previous years. This indicates that notifications of new HIV cases were affected by unstable political situation and conflicts in the country during these years $[12,13]$.

On the other hand, till the end of 2012, the cumulative number of PLHIV who continued receiving HIV treatment and care services in Yemen was 1,339, of them 721 were taking ART. Nevertheless, the number of LTFU is still increas- ing [14]. Therefore, the aim of this study is to examine demographic data of PLHIV who were enrolled in HIV treatment and care services from 2007 to 2012 and were LTFU, in order to develop rapid and effective interventions to improve access to HIV treatment and care services in Yemen.

\section{Material and methods}

The methodology is based on a retrospective descriptive study of health service data, and literature review focused mainly on demographic data for PLHIV who were LTFU for the period 2007-2012, as a baseline for future study. The study population corresponds to a group of 1,586 patients registered at pre-antiretroviral period (pre-ART) and ART period at HIV treatment and care sites in five main governorates (Sana'a, Aden, Taiz, Hodeida, and Mukalla) since 2007 till December 2012. Of them, 995 were eligible to ART, with exclusion of 170 transfer cases and 250 deaths.

Specific forms were designed to collect data. One form was designed for pre-ART patients that was registered at HIV treatment and care clinics and were not eligible for ART, while the other form was for ART registered cases. Data analysis was done by using excel sheet.

\section{Results}

\section{Data on lost to follow-up in pre-antiretroviral period}

The cumulative number of PLHIV who were enrolled in HIV treatment and care since the start of services in 2007 till the end of 2012 was 1,586; from this number, 305 patients were LTFU in pre-ART period (90\% Yemenis, $65 \%$ males). The year of 2011 obviously showed more LTFU in care as shown in Table 1.

In addition, most of LTFU in care were among married (59\%) and males (65\%), and they were mostly from governorates where HIV treatment and care services exist (Figure 1). Also, the high percentage of LTFU among females in Taiz, Sana'a, and Aden as shown in Figure 1, should be taken into consideration. Moreover, $71 \%$ of PLHIV who were LTFU in care were among the age group of 25-49 years.

Table 1. Lost to follow-up by year

\begin{tabular}{l|c|c}
\hline Year & Males (\%) & Females (\%) \\
\hline 2007 & 13 & 5 \\
\hline 2008 & 8 & 4 \\
\hline 2009 & 8 & 6 \\
\hline 2010 & 10 & 12 \\
\hline 2011 & 17 & 7 \\
\hline 2012 & 8 & \\
\hline
\end{tabular}




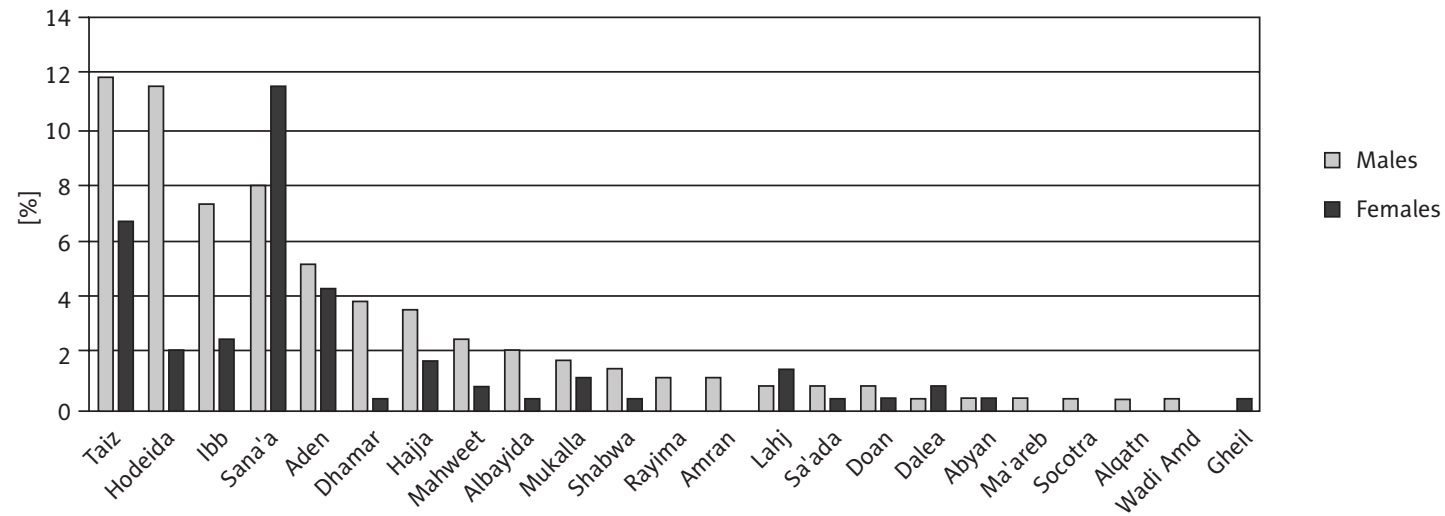

Figure 1. Patients lost to follow-up prior to initiating of an antiretroviral therapy as per a governorate

\section{Data on lost to follow-up in antiretroviral period}

Since the start of HIV treatment and care services in 2007 till the end of 2012, the cumulative number of PLHIV under ART was 995, of them 145 were LTFU (81\% Yemenis, 68\% males).

The number of PLHIV who were LTFU in ART was higher in 2011 and 2012, comparing with the previous years as shown in Figure 2.

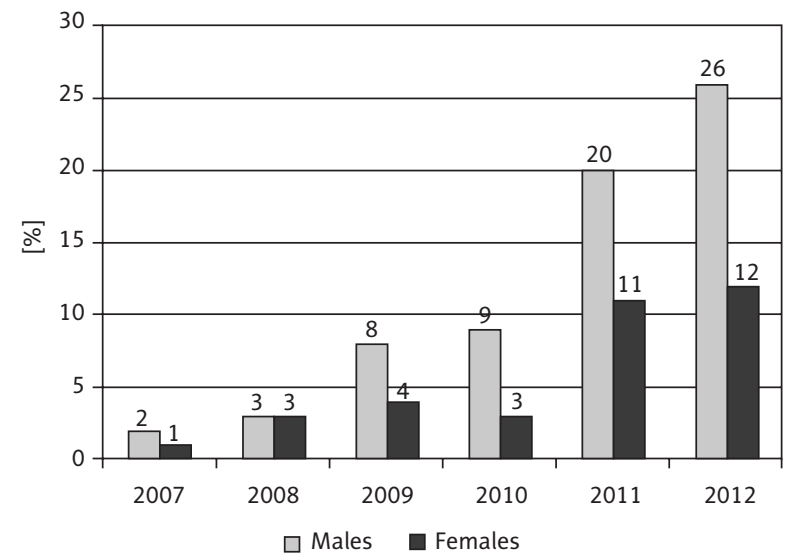

Figure 2. Patients lost to follow-up during an antiretroviral therapy by year
The LTFU on treatment were prominent among married and unmarried males as shown in Figure 3. Also, LTFU patients were mostly from governorates where HIV treatment and care sites exist, and there was high percentage of LTFU among females in Aden and Sana'a (Figure 4). The age group 25-49 year still showed higher percentage of LTFU (Figure 5). Moreover, the assessment pointed out that most of LTFU for PLHIV who were enrolled in treatment period were between the first six months to the first 3 years as shown in Table 2 .

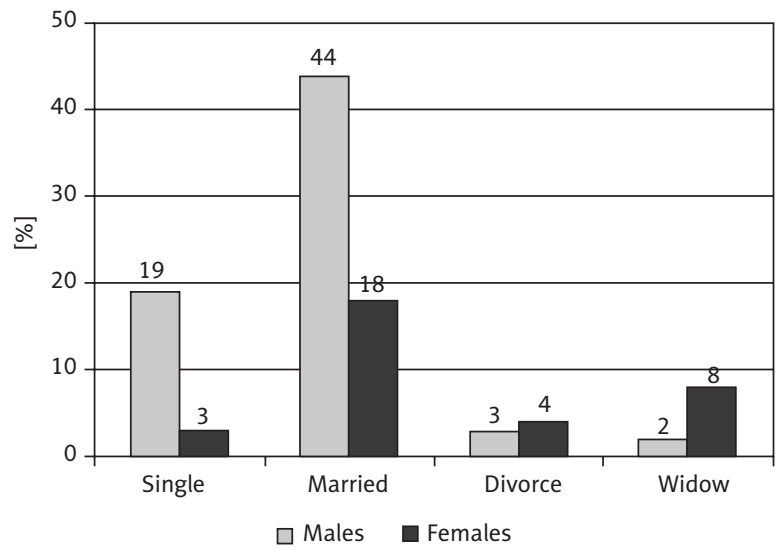

Figure 3. Marital status of patients on antiretroviral therapy that are lost to follow-up

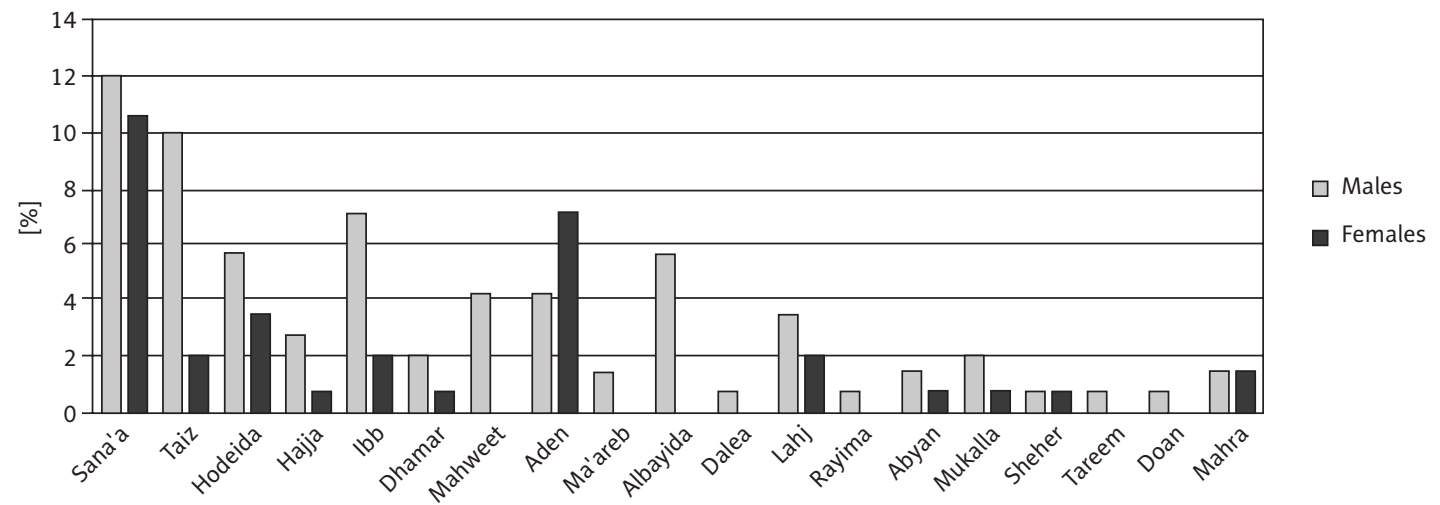

Figure 4. Patients lost to follow-up during an antiretroviral therapy as per a governorate 


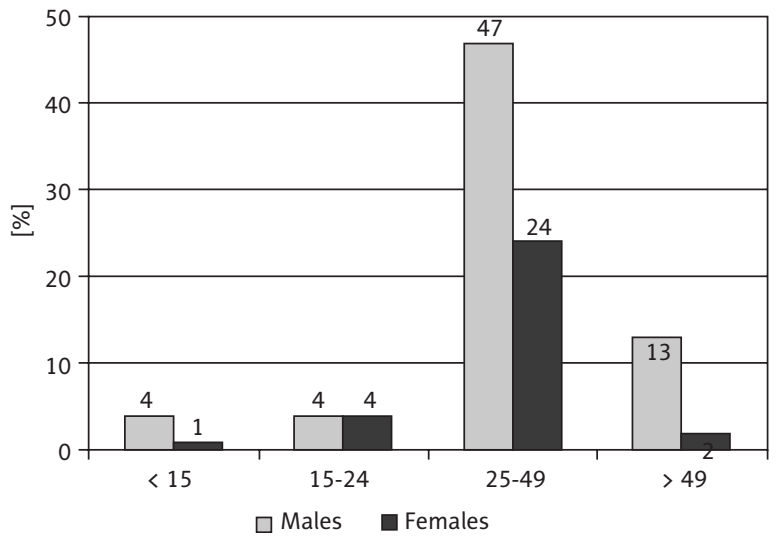

Figure 5. Patients lost to follow-up during an antiretroviral therapy by age group

\section{Discussion}

There are many PLHIV who still do not know their HIV status. PLHIV are LTFU in the period between HIV diagnosis and initiation of ART. It is recognized that most of LTFU of PLHIV in care, especially in the pre-ART period, is a major cause of poor HIV care program performance, and leads to increased morbidity and mortality. LTFU in the pre-ART period is likely because of the lack of structure and services provided for PLHIV in this 'waiting period', apart from opportunistic infection screening and prevention. Usually, the focus is on LTFU of PLHIV who are under ART, but there is no clear definition for PLHIV who LTFU at pre-ART [15]. While LTFU in ART period is define as an interval of 90 days or more, where PLHIV have missed their appointments at the ART clinic after last visit, as explained in WHO global consultative meeting report on retention in HIV care [16].

Limited HIV treatment and care services in Yemen as well as stigma and discrimination have played a role in increasing number of PLHIV who were LTFU, since the start of services [5]. The limited HIV treatment and care services to only five hospitals in five main governorates (out of 22) have hindered PLHIV access the services [7]. In addition, conflicts started mainly in main governorates in Yemen, where HIV care sites exist and experienced high number of LTFU, comparing with other governorates. However, PLHIV who are living in other governorates that have not been in conflicts, they have not been able to access HIV care services in conflict-affected governorates [7, 17].

This study has shown significant levels of LTFU patients in the pre-ART and ART periods. But most of the LTFU's were in 2011 and 2012. This might be associated with insecurity and unstable political and economic situations in the Country, where Yemen was among the countries involved in the Arab Spring revolutions [18]. Similarly, the civil war in South Sudan affected HIV care services and led to an increased number of LTFU among PLHIV [19].
Table 2. Relation between lost to follow-up and duration of enrolment in HIV treatment

\begin{tabular}{l|c}
\hline Follow-up period & Percentage (\%) \\
\hline Less than 6 months & 28 \\
\hline 6 months to 1 year & 35 \\
\hline 1 year to 3 years & 32 \\
\hline More than 3 years & 5 \\
\hline
\end{tabular}

The study reported that married and unmarried young males have higher incidence of LTFU. In contrast, the study from Asia-Pacific region and case control study conducted in Ethiopia has examined the socio-demographic and clinical factors associated with LTFU during the pre-ART period, and found that young males gender and being unmarried are associated with increased preART LTFU [15, 19]. Although, the LTFU rates among males was higher than females, governorates of Sana', Aden, and Taiz experienced highest percentage of LTFU patients in treatment and care among females, as shown in this study. This might be due to socio-cultural factors that prevent women to disclose her HIV status, and to access treatment and care services. It could also be due to political instability in those cities, since 2011, which affected females, and their access to HIV treatment and care sites [12]. Similar observation has been reported in a study conducted in Tanzania, where a large proportion of LTFU was among women [21].

LTFU frequently occurred among PLHIV who had shorter history of HIV infection [20]. In Tanzania, the LTFU occurred earlier after initiation of ART [21], which corresponds to findings in Yemen. This might be due to improper counseling given to the PLHIV at the start of ART and during each visit. Also, political conflicts led to forced displacement of population, which resulted in inability to receive good counselling. This defer adherence to treatment and may increase drug resistance [22].

The study recommends to conduct in-depth study to recognize the factors behind LTFU of PLHIV in Yemen. Also, it is important for National AIDS Control Program together with ART coordinators at governorates level to improve patients tracking system and consistent counselling. It is also recommended to develop a contingency plan that aims to overcome challenges, and improve access to HIV treatment and care services in Yemen during unstable political situations.

\section{Conclusions}

HIV treatment and care services are limited to only five main governorates in Yemen (out of 22) that experienced conflicts in 2011. Therefore, insecurity and political instability situations were responsible for most of LTFU cases that hindered access PLHIV to HIV treatment and care services in Yemen. 
PLHIV who inhabits the areas of political conflicts, especially in Sana'a, Taiz, and Aden governorates, showed a higher number of LTFU among young male groups more than in other areas located far from conflicts. Although there were LTFU in other governorates where did not experience conflict, the restricted movement of PLHIV prevented their access to HIV treatment and care services in conflict governorates. Also, the study showed a large number of females who have lost their follow-up in conflict areas.

\section{Acknowledgments}

I wish to thank all the staff at HIV treatment and care program, NAP Focal Points at governorates level and National NAP Team at central level for the efforts that have been made in data collection.

Also, I would like to thank my Professors at International Center for Casemix and Clinical Coding (ITCC), Faculty of Medicine, UKM, who guided me to publish this paper.

Project Code: FF-2017-125.

\section{Conflict of interest}

The authors declare no potential conflicts of interest with respect to the research, authorship, and/or publication of this article.

\section{References}

1. UNAIDS. Fact Sheet 2016, Global Statistic - 2015. 2016.

2. Chi BH, Yiannoutsos CT, Westfall AO, et al. Universal definition of loss to follow-up in HIV treatment programs: a statistical analysis of 111 facilities in Africa, Asia, and Latin America. PLoS Med 2011; 8: e1001111.

3. WHO. Retention in HIV programmes: defining the challenges and identifying solutions. AIDS DoH; 2011.

4. Schilkowsky LB, Crisostomo-Portela M, de Castilho Sá M. Factors associated with HIV/AIDS treatment dropouts in a special care unit in the City of Rio de Janeiro, RJ, Brazil. Rev Bras Epidemiol 2011; 14: 187-197.

5. NAP. National Strategic Plan for HIV and AIDS 2014-2018. NAP, 2015; 53.

6. NAP. National Consolidated Guidelines on the Use of Antiretroviral Drugs For Preventing and Treating HIV Infection. P. H. C. S. National AIDS Control Program, Disease Control \& Surveillance. Sana'a, Yemen. WHO, Yemen 2014; 158.

7. NAP. Country Progress Report 2012-2013, Yemen. NAP, 2014; 46.

8. CSIS. Risks and Instability in the Middle East and North Africa in 2016. CSIS, 2016.

9. OCHA. 2016 Humanitarian Needs Overview, Yemen. OCHA 2015; $1: 39$.

10. OCHA. State of Crisis: Explosive Weapons in Yemen. OCHA, 2015.

11. IOM. HIV in Emergenties. International Organization for Migration. IOM, 2014; 2.

12. Council HR. Report of the High Commissioner on OHCHR's visit to Yemen. 2011.

13. IPC. Acute Food Insecurity Current Situation Overview. Republic of Yemen. IPC, 2016.

14. NAP. National AIDS Control Program, Annual Report 2012, Ministry of Public Health \& Population, Sana'a-Yemen. NAP, 2012.
15. Ahmed I, Gugsa ST, Lemma S, et al. Predictors of loss to follow-up before HIV treatment initiation in Northwest Ethiopia: a case control study. BMC Public Health 2013; 13: 867.

16. WHO. Retention in HIV programmes: defining the challenges and identifying solutions. WHO, 2011; 71.

17. IOM. Yemen - Migration Crisis Operational Framework (MCOF) 2017-2018. IOM, 2017.

18. Group IC. Yemen at War. Sanaa/Brussels, 2015.

19. Odongkara G, Kihara C, Johnson J, et al.; Alliance IHA. The HIV response in conflict: Lessons learnt from South Sudan. 2014.

20. Zhou J, Tanuma J, Chaiwarith R, et al. Loss to Followup in HIVInfected Patients from Asia-Pacific Region: Results from TAHOD. AIDS Res Treat 2012; 2012: 375217.

21. Williams HM, Filbert F, Mmbando BP, et al. Lost to follow up and clinical outcomes of HIV adult patients. Tanzania Journal of Health Research 2012; 14; doi: http://dx.doi.org/10.4314/thrb.v14i4.3.

22. Mendelsohn JB, Schilperoord M, Spiegel P, et al. Adherence to antiretroviral therapy and treatment outcomes among conflict-affected and forcibly displaced populations: a systematic review. Conflict and Health 2012; 6: 9. 